\title{
Temps agricoles : une autre culture du temps
}

\section{Anne Moneyron}

\section{OpenEdition}

Journals

Édition électronique

URL : http://journals.openedition.org/ere/5179

DOI : $10.4000 /$ ere. 5179

ISSN : 2561-2271

Éditeur

Centr'ERE

Référence électronique

Anne Moneyron, «Temps agricoles : une autre culture du temps », Éducation relative à l'environnement [En ligne], Volume 4 | 2003, mis en ligne le 14 septembre 2003, consulté le 13 juin 2020. URL : http:// journals.openedition.org/ere/5179; DOI : https://doi.org/10.4000/ere.5179 


\title{
Temps agricoles : une autre culture du temps
}

\author{
Anne Moneyron
}

1 Dans une société qui s'interroge sur ses liens avec une nature dont elle s'éloigne de plus en plus, une société pour qui l'environnement passe de la seule notion de contour d'objets sans âme à un projet de société aux dimensions planétaires, certaines affirmations quant à l'éducation, à la notion de développement, à la ruralité au Nord et au Sud doivent être interrogées de façon plurielle. En France, dans les années soixante, les agriculteurs sont incités à devenir des chefs d'entreprise au même titre que les industriels. Sans tenir compte de la structure particulière de type familial de l'exploitation agricole, de la diversité des territoires et des savoirs qui y étaient intimement rattachés, l'agriculture fut modernisée. Aujourd'hui cette modernité n'est, nous dit-on, plus de mise. C'est le " développement durable " qui est prôné, développement de qui et de quoi ; développement pour qui et pour quoi ? L'introduction de la notion de "durabilité » peut-elle ralentir la course au développement ? Va-t-elle se résumer à maintenir le bien- être de certains êtres humains et demeurer « anthro- pocentrée » ou va-t-elle réorienter le développement sur la vie en général pour devenir " écocentrée » et ouvrir sur une prise de conscience du caractère vital et universel qui unit l'humanité et la Terre?

2 Après avoir demandé aux agriculteurs de produire sans compter, sans compter leurs efforts et leurs sacrifices culturels, financiers et humains (Salmona, 1994), il leur faut être les gardiens du paysage et du patrimoine rural. Avec la mise en place de « fermes pédagogiques ", d'un " accueil à la ferme " et autres supports, les agriculteurs sont sollicités pour devenir un temps et un espace d'éducation à l'environnement, un conservatoire pour les citadins, voire même pour certains ruraux. Mais cette nouvelle perspective du « développement » ne concerne pas tous les agriculteurs!

3 Ceux qui peuvent répondre à ce regain d'intérêt des urbains pour les campagnes, sont ceux qui sont restés, « contre vent et marées » dans une certaine logique de production extensive. Qu'ils soient agriculteurs depuis plusieurs générations ou néo-ruraux, sur des petites fermes de montagnes ou dans des zones désertifiées, ils ont voulu et ils ont 
su poursuivre leur travail selon des techniques étroitement liées à la terre et au temps des saisons, sans pour autant refuser une certaine modernité.

Quand pour la mise en place d'une agriculture « durable ", leurs savoirs deviennent à nouveau d'actualité, il ne s'agit pas alors d'en faire simplement le répertoire et l'inventaire. Parce qu'ils sont enfouis dans les mémoires, dans les terrasses agricoles, dans les sillons, les chemins de l'eau, les murets détruits qui maintenaient un équilibre écologique, il devient urgent de leur redonner vie et perspective par le prolongement d'une activité porteuse de travail et de ressources.

\section{Échapper au monopole du savoir savant}

Dans les années 90, une formation de « berger/vacher transhumant pluriactif » est mise en place par l'Association pour la formation en milieu rural (AFMR) à la demande des éleveurs locaux pratiquant la transhumance.

5 Le problème de la garde des troupeaux durant l'estive se pose de façon de plus en plus aiguë pour les éleveurs transhumants. Autrefois, la main d'œuvre était abondante dans les fermes et " il y en avait toujours un pour faire le berger " ${ }^{1}$. Aujourd'hui, là où toute une communauté familiale travaillait, une exploitation se limite souvent à une personne voire deux, ce qui ne permet pas de rester en montagne durant toute l'estive. De plus, cela génère, durant toute la saison estivale, des va-et-vient entre l'estive et l'exploitation qui sont souvent sources de perte de temps mais surtout de fatigue. Des solutions de dépannage sont trouvées mais elles sont rarement satisfaisantes. Cela va du stagiaire gratuit à une main-d'œuvre qualifiée avec si possible une expérience de la transhumance ; mais il faut noter que la grande majorité de ces bergers occasionnels n'est pas issue du monde rural et encore moins du monde agricole, ce qui pose un problème supplémentaire. Au-delà de l'apprentissage d'un savoir, ce sont les dimensions culturelles liées à une tradition « expérientielle » incontournable qu'il leur faut acquérir.

6 Ce problème de manque de main d'œuvre qualifiée fait que des estives ne sont plus occupées durant la saison d'été, ce qui pose des problèmes économiques et écologiques liés au non-pacage de la montagne par les troupeaux. Économiques car l'élevage de montagne et de certaines micro- régions, comme la Crau en Provence, a en effet besoin de ces espaces naturels pour organiser ses systèmes de production agricole. La montagne est le prolongement direct de l'exploitation agricole. Écologiques car l'absence de troupeaux dans certaines montagnes se caractérise par une sensibilité accrue aux risques naturels (avalanches) et anthropiques (incendies de forêt).

\section{Mise en place d'actions de formation}

7 La mise en place par l'AFMR et la Commission Syndicale de Soule d'une formation pour trois bergers salariés en vue d'une embauche dans trois cayolars ${ }^{2}$ a permis :

- de poser les problèmes de la mise en place d'un module de formation théorique adapté à cette activité ;

- de mieux cerner la « clientèle " potentielle du métier de berger transhumant. À priori, ce sont plutôt des jeunes gens ayant eu un contact indirect avec l'agriculture et n'ayant pas la perspective de reprendre une ferme ; 
- de mieux définir les besoins des éleveurs en matière de gardiennage, besoins qui semblent plus pressants en Béarn qu'au Pays Basque ;

- de dégrossir les problèmes concernant le statut juridique et fiscal des bergers salariés. dans leur recherche de « main-d'cuvre compétente et qui reste! ", la décision est prise de poursuivre la formation tout en contribuant à sa lisibilité en terme de savoir et à sa pérennisation en terme de métier. C'est à cette époque le cadre du dispositif des « nouvelles qualifications » qui est choisi. Ce dispositif vise à aborder les problèmes liés à la création de nouveaux métiers ou à la rénovation de métiers anciens, afin d'éviter les risques de sur- qualification ou de sous-qualification liés à une mauvaise définition du métier et à une mauvaise coordination entre employeurs, employés et centres de formation. La démarche des «nouvelles qualifications » fut initiée en 1984 par Bertrand Schwartz, alors délégué interministériel à l'insertion professionnelle et sociale des jeunes en difficulté. En 1989, elle est élargie au public adulte.

L'action de formation intitulée " berger/vacher transhumant pluriactif » est plus spécifiquement une formation-développement qui s'articule autour de quatre principes :

- l'acquisition de compétences se fait selon le principe d'une alternance inversée : le lieu de travail est reconnu comme lieu principal d'acquisition des savoirs ;

- une réflexion sur l'organisation du travail est menée en même temps que la formation proprement dite afin de permettre une insertion professionnelle stable de la personne en formation;

- elle s'adresse à des personnes sans qualification ou avec un bas niveau de qualification;

- le partenariat (AFMR, Mission locale, Centre ovin) sert de support à la réflexion sur l'évolution du métier, les problèmes de sa pérennisation, la question de l'identification des savoirs et de leur validation tant sur le terrain qu'en centre de formation.

\section{Premier essai de mise en forme du savoir des bergers transhumants}

En 1989, à partir de réunions réalisées avec des bergers et des éleveurs, tuteurs de l'action de formation, une première écriture des savoirs est ébauchée et sert de support à la formation des apprentis. Elle s'avère peu satisfaisante car trop centrée sur les savoirs techniques essentiellement liés à la manipulation des animaux et à la conduite sanitaire du troupeau, c'est-à-dire le travail d'un berger ou d'un éleveur ovin en plaine.

11 Ayant les mêmes interrogations que le " groupe recherche " déjà au travail, nous prenons connaissance de cet écrit avec le souhait de comprendre, dans le sens de prendre ensemble (con-prehendere) ces savoirs spécifiques à la transhumance. À la relecture, la dimension " estive », en tant que lieu d'acquisition d'un savoir particulier et lieu de confrontation des références culturelles propres à chacun qu'il soit ancien ou apprenti berger, apparaît immédiatement comme absente. Les " anciens » eux-mêmes nous disent de cette transcription de leur métier, « berger, ce n'est pas que ça! ».

Mais ce «ça!", c'est quoi ? Existe-t-il un savoir spécifique à l'estive ? Comment faire pour échapper au monopole du savoir savant ? Peut-on apprendre autrement, autre chose ? Comment valoriser et faire reconnaittre ce savoir non officiellement reconnu?

Une intuition couplée à l'expérience personnelle fait ressentir que ce « ça!» se trouve ailleurs et qu'il n'est pas exprimable dans une salle de réunion. Il faut se libérer de ces réunions/échanges où le corps n'a pas sa place, où les yeux sont constamment rivés aux

Éducation relative à l'environnement, Volume 4 | 2003 
fenêtres pour voir l'évolution du temps parce que le travail est là-haut. Il faut retrouver l'espace d'un autre rythme qui ouvre sur le geste de l'estive et permettre de " passer des regards croisés aux regards partagés " (Laplantine, 1996, p. 16). Avant de pouvoir parler d'apprentissage et de transmission du savoir, il nous faut trouver ensemble un accord sur la nature de ce "ça!». Nous acceptons de tenter de comprendre et d'identifier ce savoir. Pour cela, au rythme de plusieurs aller-retour entre la montagne et la plaine, au cours des différentes périodes d'été et d'hiver, dans des lieux éclatés tels le centre de formation, les estives, les marchés, nous rencontrons des apprentis, des tuteurs et des responsables de structures agricoles, tous concernés par la formation. Ce qui conduira dans un premier temps à un essai d'identification de la nature spécifique du savoir puis, dans un deuxième temps, à percevoir comment son passage est rendu possible entre un ancien et un apprenti.

\section{L'expérience de la peur et la formation du savoir transhumant}

14 L'estive oblige à réfléchir à la transmission, à l'identification et à l'acquisition du savoir transhumant selon une logique particulière car « il existe des principes naturels encore plus forts que ceux qui régissent nos sociétés » (de Rosnay, 1995, p. 18). En partant des propos des bergers rencontrés en montagne, il est possible de discerner une dimension commune à leurs expériences : celle de la peur et du risque, expériences qui demandent la mobilisation de tous les sens afin de s'adapter à un nouveau quotidien de travail et de vie. Peur liée à un univers inconnu que l'on ne peut maîtriser, inhérente à la situation de travail, et que l'on aborde dans la solitude de l'estive, sans médiateur humain. Les apprentis bergers osent encore exprimer leurs peurs, tandis que les anciens parlent de la prise de risque. Ainsi, pour un apprenti qui n'est pas " nourri " du savoir transhumant, c'est la transformation personnelle de l'expérience vitale de la peur qui va le conduire au degré de vigilance indispensable. Cette transformation demande de comprendre le troupeau autrement que par une vision technique et anthropocentrique.

\section{Un savoir qui se construit avec l'environnement}

15 Les témoignages des bergers rencontrés en estive montrent comment la place de l'animal s'avère dominante dans la construction du savoir transhumant. Les personnes (tuteurs, formateurs) au cours de l'apprentissage et de la pratique du métier semblent avoir une part congrue. Dans cet environnement particulier de l'estive, qui se décline au gré du rythme, des repères et des périodes de l'estive, le temps du berger et le temps du troupeau semblent être uniques.

16 Le premier des temps évoqués, c'est le rythme, «le berger, il a son rythme, il ne marche pas comme tout le monde, il prend son temps». Étymologiquement, le rythme signifie « ce qui coule, dans le temps ou dans l'espace " (Brunet et al., 1992, p. 400). Le rythme des bergers est à la fois un et multiple. Son rythme personnel doit se régler sur celui de son troupeau. Pour l'apprenti berger, c'est une course contre sa fatigue, «c'est vrai que pour gratter une demi- heure de sommeil, il va monter comme un dératé pour redescendre se recoucher à la cabane [...] le jeune, il peut pas faire la sieste dehors "; tandis que le rythme du berger expérimenté est posé, "faire la sieste sur le coup de 8 heures, une fois que tu as envoyé les bêtes [...] et puis des fois je m'endors carrément, il est 11 heures, le soleil de me 
réveiller, je vois qu'elles sont toutes là et je redescends à la cabane ". Non seulement l'apprenti berger va vite pour pouvoir se reposer parce qu'il a de la difficulté à régler son rythme avec celui du troupeau qui, quoiqu'il arrive démarre au lever du jour, mais " il ne peut pas faire la sieste dehors ", ce qui l'amène à un surcroît de fatigue dû à d'inutiles aller-retour vers la cabane. L'apprenti berger doit régler son rythme avec celui du troupeau sinon il s'épuise pour rien. Une pratique en force et à contretemps du métier n'est pas possible à long terme, car " on ne gagnait rien à résister à l'environnement, et qu'il valait mieux au contraire, s'y adapter, s'y fondre » (Hall, 1984, p. 53.)

17 Viennent ensuite les repères. Il est intéressant de noter que le mot repère, qui veut dire la marque ou le signal indiquant un emplacement, a la même origine étymologique que le mot " repaire » au sens d'habitat sauvage, avec l'idée que le repère permet de se retrouver chez soi.

Le repère sert soit à se situer, à s'orienter [...] se retrouver dans l'espace ; soit comme base d'évaluation d'une distance, y compris dans le temps ; soit pour indiquer un risque, un danger. Tout en fixant, le repère donne des formes de liberté : celle qui permet de vaincre l'étendue. (Brunet et al., 1992, p. 387)

À travers ces trois approches du mot repère, nous retrouvons les éléments mentionnés par les bergers. Le repaire, tel la cabane, est à la fois sécurisant car il permet de se retrouver un peu chez soi, de s'approprier ce nouvel espace, « au jeune je lui dis : quand $i$ fait du brouillard tu restes à la cabane [...] et tu attends ». Le repère est sécurisant aussi parce qu'il donne une indication sur les dangers dans le temps et dans l'espace. En montagne, les repères sont communs à tous les utilisateurs, chaque point remarquable est un code, a un nom, chargé de l'histoire de la montagne, de l'histoire des bergers et des troupeaux. Histoires qui se transmettent, " je crois que quand on est nouveau en montagne, faut pas avoir peur de se faire expliquer ». Mais se former à l'estive signifie que chacun doit se faire ses propres repères, " il faut se faire ses repères [...] découvrir tous les chemins, tous les passages ", afin d'accéder à une certaine liberté de travail et se fondre sans se confondre dans la « vastitude » de la montagne.

Les périodes de l'estive sont celles du cycle, du climat et de l'instant. Ces trois éléments ont en commun d'être ceux d'un espace-temps, support des formes de l'alternance vitale qui se déroule au cours des mois de l'estive.

Le cycle est l'alternance naturelle de phénomènes se renouvelant selon un ordre immuable avec, contrairement au rythme, la notion de boucle, de retour au point de départ. Ce sont le jour et la nuit, les aurores et les crépuscules. Le cycle biologique des animaux et le cycle des saisons sont étroitement liés l'un à l'autre. Ces cycles sont les supports de l'organisation de l'estive, du parcours choisi par et pour les bêtes pour une alimentation optimale en herbe.

21 Dans les conversations, plusieurs situations critiques liées au climat, ce temps qui enveloppe le berger et contre lequel il ne peut rien, mais avec lequel il doit composer en permanence. Il lui faut prévoir l'orage, pour lui et son troupeau. En montagne, les accidents dus à la foudre ne sont pas exceptionnels, parfois mortels pour le berger et,ou quelques-unes de ses bêtes. Dans ces situations électriques, le berger doit anticiper, réagir très vite, au bon moment, pour faire descendre son troupeau de la crête parce que « les brebis quand il y a de l'orage, il faut pas les emmerder ». Et puis il y a le brouillard, moins critique pour le troupeau qui, selon les estives et s'il est laissé tranquille et libre de ses mouvements, va simplement se mélanger avec d'autres bêtes. Le brouillard, c'est 
surtout difficile à vivre pour l'apprenti berger, qui doit apprendre à ne rien faire, «ça arrive de se perdre dans le brouillard, il faut savoir que quand on est perdu, on se couche et on attend le lendemain; en été on ne risque rien ».

Enfin l'instant est « le temps de l'espace d'un pas » (Dictionnaire Hachette). Le pas du berger pris comme référence d'espace et de durée est celui qui, quand il est tranquille, rythme la journée mais quand il est plus rapide, il rythme les interventions. Ce pas, selon le temps et l'espace qu'il recouvre, est bon ou mauvais, «le berger de St-Paul, lui, s'il est mort, c'est qu'il s'est pas trouvé quand il fallait, ou il fallait ».

\section{Un savoir imprégné de la réalité quotidienne}

Le savoir du berger transhumant, entre nature et culture, est formé par un rapport permanent avec une réalité qui n'appartient plus au quotidien d'une société de plus en plus urbanisée ; il est réalité du mouvement d'une sensibilité écologique. En montagne, tout incident physique ou moral peut avoir des conséquences graves, voire mortelles, en cela « le savoir-faire transhumant relève de celui d'un montagnard " (Duclos, 1994, p. 17). Mais si le savoir du berger n'est pas celui de l'éleveur, ce savoir ne se limite pas non plus à celui d'un montagnard car le berger est avant tout le gardien responsable d'un troupeau. Et c'est en observant cette relation écologique avec les choses, à travers le savoir des bergers transhumants, que nous pouvons approcher le fondement vital de la forme humaine. Il s'agit de réintégrer le réel dans nos questionnements, d'aller voir " dans les sociétés autre chose que des relations humaines » (Sigaut, 1990) et de ne plus " ignorer un réel incommode » (Sigaut, 1990), d'ouvrir sur une réalité qui inclut à la fois le réel et l'imaginaire. La réalité pratique peut alors être réfléchie comme " un pli de l'ensemble des niveaux de perception » (Nicolescu, 1996, p. 105), là où « le réel est un pli de l'imaginaire et l'imaginaire est un pli du réel » (Nicolescu, 1996, p. 105).

\section{La transhumance, nécessité d'une trans-formation}

Transhumer, cheminer, sont des temps, des espaces, des parcours singuliers qui suivent des itinéraires inconnus sur lesquels les repères sont à faire ou à découvrir, afin de s'ouvrir à son propre chemin. Ce n'est qu'à cette condition, en prenant et en acceptant le risque de sortir de ses propres chemins battus, que «l'homme grandit en densité, en allant de sortie en sortie " (Santaner, 1992, p. 20). Si transhumer est le mouvement propre à la vie, ce mouvement n'est pas Vie en soi. Pour qu'il y ait vie, au-delà du mouvement, il faut qu'il y ait sortie, sortie de soi, sortie de l'environnement connu qui rassure et protège.

Pour redécouvrir cette expérience incorporée, pour lui laisser prendre sa forme et sa place, il faut se déplacer. L'espace de la transhumance se réfléchit essentiellement en simple question de vie et de mort, " comme je dis toujours, le berger part seul en montagne, sans dire où il va, donc déjà au départ [...] il prend des risques ». L'estive, c'est l'école de l'aléa qu'il faut découvrir et baliser avec le troupeau, avant de ne devenir fou comme certains en témoignent et avant de prendre des risques inutiles ; mais c'est aussi une école où l'on se transforme à l'écoute sensible de l'environnement, où « le temps de travail ne se partage pas en heures, mais se calque sur le rythme du vent qui « pousse » ou qui « calme » » (Delbos et Jorion, 1990, p. 193). 


\section{Nommer le savoir des transhumants : éco-savoir de la présence}

Si ce savoir n'est ni pratique ni théorique, s'il est rapport au monde, alors comment sortir d'un découpage technique et social du travail selon l'approche ternaire du savoir/savoir-faire/savoir-être et s'interroger sur ce rapport pratique au monde. La clef qui permet de nommer ce savoir du contact et de la relation intime avec la nature est à la base des réflexions et des travaux de Gaston Pineau en ce qui a trait à « l'écoformation ".

L'étude des propos, des gestes recueillis et l'approche de la formation de Gaston Pineau permettent de nommer ce savoir spécifique aux transhumants. Gaston Pineau par sa théorie tripolaire de la formation revitalise la théorie éducative de Jean-Jacques Rousseau en trois pôles de la formation expérientielle : « auto-soi, éco-les choses, hétéro-les autres » (Pineau, 1986, p. 146). Il redonne une place à part entière aux choses qui animent notre quotidien, qui nous forment, nous transforment. Si la formation autoréférencée est étudiée depuis plusieurs années, il n'en va pas de même pour l'écoformation. C'est par son travail sur " un élément-milieu particulier : l'air » que Gaston Pineau (1992) ouvre ce champ de recherche qu'il nomme l'écoformation. Dans son sillage, avec sa recherche et sa pratique des classes de mer, qu'elle souhaite plutôt comme une école des éléments, Dominique Cottereau (1994) propose une pédagogie de l'imaginaire et de l'écoformation.

Cette pédagogie « se voudrait conscientisation du lien écologique que nous avons avec la nature, connaissance pour une éco-naissance » (Cottereau, 1994, p. 123).

Au cours de la transhumance, le troupeau et la montagne renvoient à des temps et des espaces de relations avec l'animal, le végétal et le minéral, qui transforment les formes écologiques d'une alternance bio-cognitive en un éco-savoir vital. La réflexion entamée en estive à propos de l'homme au travail avec la nature, se veut celle d'une éconaissance qui repose sur l'expérience de l'attention en tant que présence à l'autre. Pour développer une autre relation avec un environnement inconnu, pour être capable physiquement "d'être là où il faut et quand il faut ", l'apprenti doit d'abord passer par un " dés-apprentissage, ce désapprentissage peut requérir un entraînement et un effort, mais il s'agit d'un effort différent de celui qui nécessite l'acquisition de quelque chose de nouveau » (Varela, 1996, p. 61). Dans la solitude et le silence de l'estive, le « simple fait d'être-là, dans le faire- face immédiat, est loin d'être purement et simplement une question de " réflexes " (Varela, 1996, p. 37). Cette expérience solitaire de l'environnement ouvre à l'immédiateté (Varela, 1996) et c'est par la grâce de ce temps suspendu que s'exprime "le wu-wei/rien-faire qui désigne une expérience et un parcours d'apprentissage, et non une simple découverte intellectuelle " (Varela, 1996, p. 56). Plus qu'un savoir pratique, c'est bien un éco-savoir qui est incarné et mis en œuvre dans le mouvement de l'instant où, "lorsque l'action non-duelle se déroule régulièrement, l'acte est ressenti comme fondé dans ce qui est calme et ne se meut pas » (Varela, 1996, p. 59). En effet, avec le concept d'écoformation, nous nous situons dans une nouvelle perspective de la formation. D'une part, celle d'une relation inverse où l'apprenti se forme par les choses et d'autre part, celle d'une relation où les choses forment l'apprenti. Une écoformation dont les bergers témoignent en ces termes « il 
m'arrivait de me demander si je gardais bien les brebis ou si c'était elles qui me gardaient » (Melet, 1978, p. 154).

\section{Les temps agricoles : une culture « écoformée » du temps}

30 L'approche écoformée du savoir élargit la compréhension du métier par la prise en compte de l'animal en tant qu'être vivant et sensible et non plus en tant que machine à produire de la viande. La sensibilité animale n'est plus considérée comme une contrainte, l'interaction et la coordination des rythmes de l'homme et de l'animal se trans-forment en un éco-savoir bio- cognitif qui structure l'homme et sa pensée pratique, pensée concentrée sur l'efficacité du geste quotidien. De plus, la reconnaissance du savoir des bergers permet de repenser le travail avec l'animal de rente, non plus sur le mode de la domination mais selon une logique de réciprocité sensible. Par cette reconnaissance d'un éco-savoir, nous accédons à l'expression transfrontalière d'un monde qui ressent l'unité des choses.

31 La question de "l'homogénéisation d'un savoir pratique productif, selon un modèle abstrait qui évacue nécessairement toute la partie vivante " (Delbos, 2000) si elle est soulevée par cette auteure à propos du savoir des paludiers ${ }^{3}$, est également abordée par Michèle Salmona (1994) à propos des éleveurs et des maraîchers. De même Georges Rossi (2000, p. 90), dans une étude sur l'ingérence écologique, fait le même constat : « la domination de la conception occidentale de l'élevage, la certitude qu'il convenait d'améliorer un système incohérent, ont très longtemps empêché que l'on s'intéressât de près aux savoirs des pasteurs ». Au-delà de la normalisation du savoir, cette vision a tendance à déposséder les éleveurs d'une dimension fondamentale de leur savoir : l'affectivité ; elle inhibe l'action des paysans qui n'osent plus faire comme avant, de peur d'être jugés comme " arriérés ", alors que "l'adaptation permanente et le changement font partie de la vie des paysans »(Rossi, 2000, p. 99), ce que Henri Mendras (1984, p. 41), quelques années auparavant, pointait déjà : « les sociétés en apparence les plus figées sont le produit d'une longue évolution faite d'expériences, d'échecs et de progrès ».

\section{Conclusion}

L'idée évoquée à travers ce propos sur le temps dans l'agriculture est qu'il est vain de vouloir mesurer et découper le travail agricole selon les normes de la production industrielle. En effet, pour les agriculteurs en général et les éleveurs en particulier, leurs temporalités imbriquent étroitement temps de vie et temps de travail. Parce que les bêtes mangent, s'accouplent, mettent bas en fonction de leurs cycles et rythmes biologiques et non selon un calendrier ou un horaire établi une fois pour toute. Parce que pour détecter les chaleurs des animaux, il faut traîner dans les champs, dans les étables ; parce qu'au moment des mises bas, qui se passent essentiellement le soir jusqu'aux alentours de minuit et le matin dès trois ou quatre heures, l'éleveur doit être " présent » en permanence, physiquement ou mentalement, auprès de ses bêtes.

Dans notre travail de recherche, l'étude du savoir des bergers transhumants permet de pointer de façon fine cette nécessité de la présence à l'autre-animal. En étudiant le 
quotidien de leur vie et de leur pratique de travail en estive, plus précisément à partir de l'expérience de la peur commune à plusieurs générations de bergers transhumants, un éco-savoir fondamental formé de la relation à l'animal est mis à jour quand le berger transhumant règle son pas avec l'allure de ses bêtes et qu'il s'en remet au rythme de son troupeau pour ménager sa fatigue physique et donner de la précision à ses interventions. Ce savoir se constitue de deux temps essentiels : le rythme lent qui s'étire tout au long de l'estive et l'instant bref de l'intervention parfois vitale que le berger doit réaliser. Cette inscription écoformée du savoir est à la fois la motricité qui permet d'agir au bon moment, l'instant du développement du geste et la sensation indispensable à la perception, l'anticipation et l'observation, le rythme d'incorporation du geste.

Le rapport au temps n'est plus référencé selon la logique sociale d'un calendrier fixe et des aiguilles de la montre, mais en fonction des aléas qui rythment la vie, dans une relation d'altérité avec le troupeau, que le berger garde tout au cours de l'estive. Pourquoi ? Simplement parce que «tous ces devenirs sont les devenirs heureux de la lenteur » (Bachelard, 1986, p. 86).

\section{BIBLIOGRAPHIE}

Bachelard, G. (1986). La flamme d'une chandelle. Paris : Presses Universitaires de France. Brunet, R., Ferras, R. et Théry, H. (1992). Les mots de la géographie : dictionnaire critique. Paris : La Documentation Française.

Cottereau, D. (1994). À l'école des éléments : écoformation et classe de mer. Lyon : Chroniques Sociales. Delbos, G. (2000). Dans les coulisses du patrimoine. In D. Chevallier (dir.), Vives campagnes : le patrimoine rural, projet de société. Paris : Autrement, p. 97-128.

Delbos, G. et Jorion, P. (1990). La transmission des savoirs. Paris : Éditions de la Maison des Sciences de l'Homme.

De Rosnay, J. (1995). L’homme symbiotique. Paris : Éditions du Seuil.

Duclos, J.-C. (1994). L'homme et le mouton dans l'espace de la transhumance. Grenoble : Éditions Glénat.

Hall, E.T. (1984). La danse de la vie : temps culturels, temps vécus. Paris : Éditions du Seuil. Laplantine, F. (1996). La description ethnographique. Paris : Nathan.

Melet, P. (1978). Bergers mes amours. Grenoble : Didier et Richard. Mendras, H. (1984). La fin des paysans. Paris : Actes Sud.

Nicolescu, B. (1996). La transdisciplinarité. Monaco : Édition du rocher. Pineau, G. (1992). De l'air. Essai sur l'écoformation. Paris : Païdeia.

Pineau, G. (1986). Temps et contretemps en formation permanente. Paris : UNMFREO.

Rossi, G. (2000). L'ingérence écologique. Environnement et développement rural du Nord au Sud. Paris : Éditions CNRS. 
Salmona, M. (1994). Les paysans français, le travail, les métiers, la transmission des savoirs. Paris :

L'Harmattan.

Santaner, M.-A. (1992). De l'exode à l'exil. Paris : Desclée de Brouwer.

Sigaut, F. (1990). Folie, réel et technologie. Revue Techniques et Cultures, 15, 167-179. Varela, F.

(1996). Quel savoir pour quelle éthique. Paris : La découverte.

\section{NOTES}

1. Les phrases reproduites entre guillemets et en italique sont extraites de nos conversations avec les bergers transhumants en estive.

2. Le cayolar est le terme basque qui désigne à la fois la cabane du berger en montagne et l'organisation du système pastoral en estive.

3. Les paludiers sont les exploitants des marais salants qui collectent le sel de mer.

\section{INDEX}

Keywords : education, development, agriculture, sustainable development, rurality, transhumance

Mots-clés : éducation, développement, agriculture, développement durable, ruralité, savoirs profanes, transhumance

\section{AUTEUR}

\section{ANNE MONEYRON}

Consultante en formation des adultes, Anne Moneyron anime des formations-actions sur l'identification, la transmission et la formation des savoirs « écoformés » dans le quotidien de travail et de vie des « métiers du soin » dont ceux d'agriculteurs et de travailleurs sociaux par exemple. Elle est diplômée d'un doctorat en sciences de l'éducation et de la formation. 